\title{
Expression of angiogenic markers in jawbones and femur in a rat model treated with zoledronic acid
}

\author{
Jing Wen $\mathrm{Li}^{1 \dagger}$, Jing Yi Wang ${ }^{1 \dagger}$, Ru Qing Yu ${ }^{1}$, Lei Huo ${ }^{2}$ and Li Wu Zheng ${ }^{1 *}$ (i)
}

\begin{abstract}
Objectives: This study aimed to investigate the gene expression of angiogenic marker in surgically treated jawbones and femur on a rat model administrated with zoledronic acid.

Results: No soft tissue fenestration or bone exposure was found in femur. Delayed soft tissue healing was found in both ZA group ( 3 in mandible, 4 in maxilla) and control group ( 1 in mandible, 2 in maxilla), while exposed bone was found only in the ZA group (1 in maxilla, 2 in mandible). RT-PCR analysis demonstrated no significant difference in gene expression of angiogenetic markers between ZA-treated and control groups in femur and mandible. In the maxilla, the expression of VEGFA and VEGFR-2 in medium-term ZA group was significantly down-regulated compared with that in the control. The ZA treatment does not change significantly the expression of the angiogenic factors in femur and mandible, but significantly downregulates the expression in maxilla in this rat model. The angiogenesis inhibition may contribute to the development of MRONJ but does not play a key role.
\end{abstract}

Keywords: Medication-related osteonecrosis of the jaw, Zoledronic acid, Animal model, Gene expression, Angiogenic markers

\section{Introduction}

Bisphosphonates (BPs) have been extensively used for management of bone diseases with pathologically high resorption. Despite the great clinical benefits, a severe complication known as medication-related osteonecrosis of the jaw (MRONJ) has been reported [1]. The potentially compromised neovessel formation in the development of MRONJ has been investigated but the results remain controversial.

Vascular endothelial growth factor A (VEGFA) plays a critical role in angiogenesis, promotion of vessel permeability and also participates in the processes of bone tissue

*Correspondence: Iwzheng@hku.hk

${ }^{\dagger}$ Jing Wen Li and Jing Yi Wang contributed equally to this work

${ }^{1}$ Discipline of Oral and Maxillofacial Surgery, Faculty of Dentistry, The

University of Hong Kong, Hong Kong SAR, China

Full list of author information is available at the end of the article formation, healing and remodeling [2-5]. VEGFA binds to target cell receptors such as VEGF receptor (VEGFR)$1,-2$ and -3 . At binding, VEGFA activates these receptors in extracellular space (ECs) and promotes transduction of different signals in EC migration, proliferation and enhancement of angiogenesis [6,7]. VEGFR-2 is an essential mediator of VEGF-initiated angiogenesis. Levels of VEGFR-2 expression is relatively low in adult blood vessels, however, it is markedly upregulated in blood vessels in some pathological conditions, such as chronic inflammation, wound repair and cancer [8-11]. CD31 is a marker of vascularization inclusive of microvessels [12-14]. S. Ishtiaq et.al investigated the circulating concentrations of VEGF and angiopoietin-1 (ANG-1) in post-menopausal women receiving alendronate therapy, and with osteoblastic production of VEGF and ANG-1 under zoledronate and alendronate treatments in vitro. [15] The results showed that BPs suppressed osteoblastic 
production of angiogenic factors in vitro and in vivo, indicating the possible relevance of angiogenesis inhibition in MRONJ pathophysiology. However, other studies revealed an upregulation of angiogenic factors after long-term BPs treatment, which might be a result of compensation for BPs' antiangiogenic potency [16-18]. Interestingly, levels of serum markers of angiogenesis and inflammation were also found to be higher in MRONJ patients after cession of long-term BPs therapy [17]. Higher expression of VEGFA in postoperative exudate of bisphosphonate-related osteonecrosis of the mandible was also reported [19]. There are reports showed decreased expression of VEGF in oral mucosa in patients receiving BPs treatment without MRONJ lesions, and a massive increase of VEGF in MRONJ mucosal lesions [20]. The contradictory findings suggested a possible alteration of angiogenesis in the development of MRONJ. The evidence is still lacking for the angiogenic character of this pathological condition.

This study aimed to investigate the gene expression of angiogenic markers in surgically treated jawbones and femur using a rat model administrated with zoledronic acid administration.

\section{Main text}

\section{Material and methods}

\section{Animal care and surgery}

Thirty-two 12-week-old female Sprague-Dawley (SD) rat were obtained from the Laboratory Animal Unit of Li Ka Shing Faculty of Medicine, The University of Hong Kong. The protocol of the animal experiment was approved by the Committee on Use Live Animal for Teaching and Research, The University of Hong Kong (CULATR 377515). The animals were housed in an indoor environment at a temperature of $20{ }^{\circ} \mathrm{C} \pm 5^{\circ}$ in a 12:12-h light-dark circle with free access to water and standard rodent diet (Irradiated, PMI, USA).

Animals were general anesthetized intraperitoneally with a mixture of $67 \mathrm{mg} / \mathrm{kg}$ ketamine and $6 \mathrm{mg} / \mathrm{kg}$ xylazine (Alfasan International B.V., Woerden, Holland). The right thigh was shaved and sterilized with $10 \%$ betadine solution. A $1.5-\mathrm{cm}$-long incision was made followed with fascia and muscle dissection. Femer was completely exposed after periosteum exfoliation. A unicortical circular defect was created at the lateral aspect of femer with a round bur using a Ø2.0 trephine bur (Trephine Drill $2 \mathrm{~mm}(3 \mathrm{~mm}$ OD$) \times 10 \mathrm{~mm}$ Barrel, ForevreGreen, Hong Kong) by low-speed handpiece without any further invasion to bone marrow. Surgical site was continuously irrigated with sterile saline solution to avoid contamination and overheat. Cortical bone layer was then removed and bone marrow was exposed. The soft tissues were closed in two layers. The muscular layer was sutured using 4-0 vicryl (Ethicon Inc., Cornelia, GA), the skin was sutured using 5-0 Prolene (Ethicon Inc., Cornelia, GA). Following the right femur operation, the right first maxillary and mandibular molars were extracted using a standard protocol. Two gauze rolls and a retractor were used to maintain the mouth open and to make the tongue immovable. After gingiva separation with dental explorer the molars were carefully removed using children's extracting forceps, in order to avoid any damage to surrounding tissue. The extraction sites were left open and a small cotton wool roll was pressed onto the extraction socket until bleeding stops.

\section{Grouping and treatment}

Sixteen rats received zoledronic acid treatment (ZA group), and the rest rats serving as the control group received saline solution. Eight rats in each group were sacrificed at 2 weeks (short-term group) and 4 weeks (medium-term group) after surgery, respectively.

From four weeks before surgery until sacrifice, animals in ZA groups were given $66 \mu \mathrm{g} / \mathrm{kg}$ of zoledronic acid (Zometa, Novartis, Switzerland) intraperitoneally three times per week. In control group, the same volume of normal saline solution was administered with the same timing protocol.

\section{Sacrifice, sample collection and preparation}

Pentobarbital sodium (Dorminal ${ }^{\circledR}$, Alfasan International B.V., Woerden, Holland) was administered at a dose of $150 \mathrm{mg} / \mathrm{kg}$ intraperitoneally for animal sacrifice. Euthanasia was confirmed by observing that there were no respiratory movement at least three minutes and heartbeat had ceased. Gross observations of the samples were performed, and photo records were taken.

The jawbones and right femur were removed with soft tissue stripped off. A rongeur was used to collect the bone tissue in the extraction socket and femur defect. The collected tissue was immediately stored in RNAlater $^{\circledR}$ solution (AM7024, Life Technologies Limited, California, United States) and stored at $-20{ }^{\circ} \mathrm{C}$ for further assessments.

\section{RNA extraction and $C D N A$ synthesis}

The total RNA from femur defect site and jawbones tooth extraction site in both control and ZA-treated samples were extracted using RNeasy Lipid Tissue Mini Kit (QIAGEN, Hilden, Germany). The total RNA concentrations were quantified using Nanodrop ND-2000c spectrophotometer (Thermo Fisher Scientific, Massachusetts, US) at $260 \mathrm{~nm}$, and non-contamination with proteins was verified according to the 260/280 ratio. The SuperScript III Reverse transcriptase (Invitrogen Corporation, US) was used to synthesize complementary fxdeoxyribonucleic 
acid (cDNA) in a PCR thermal cycler (GeneAmp PCR System 9700, Thermo Fisher Scientific, Massachusetts, US).

\section{Real-time polymerase chain reaction (RT-PCR) analysis} Quantitative real-time PCR (RT-PCR) was performed using FAST SYBR ${ }^{\mathrm{TM}}$ Green Master Mixes (Applied Biosystems $^{\mathrm{TM}}$, Hercules, CA, USA) in ABI Prism 7000 sequence detection system (Applied Biosystems ${ }^{\mathrm{TM}}$, Hercules, CA, USA) following manufacturer's instruction.

All samples were run in MicroAmp ${ }^{\mathrm{TM}}$ Fast Optical 96-well reaction plates. The reactions conditions were performed at $95{ }^{\circ} \mathrm{C}$ for $20 \mathrm{~s}$, following with 40 cycles of $95^{\circ} \mathrm{C}$ for $3 \mathrm{~s}$ and $60{ }^{\circ} \mathrm{C}$ for $30 \mathrm{~s}$. Four pairs of primers, including CD31, VEGFA, VEGFR-2 and housekeeping gene GAPDH, were designed using Primer Express software (Applied Biosystems ${ }^{\mathrm{TM}}$, Hercules, CA, USA) (Table 1). Relative quantitation of gene expression was assessed between quantity of target gene and GAPDH using relative standard curve method.

The expression values of CD31, VEGFA and VEGFR-2 were normalized to the expression of GAPDH. A standard curve was carried out using the target DNA which subjected to a serial dilution. The signals in the sample were within the cycles covered by the standard curve. A melting curve was carried out to indicate the quality of the product, in which a high-quality product gave a narrow peak when denatured.

Table 1 Primers designed for amplifying target and housekeeping genes in RT-PCR

\begin{tabular}{lll}
\hline Gene & Forward primer $\left(\mathbf{5}^{\prime} \mathbf{-} \mathbf{3}^{\prime}\right)$ & Reverse primer $\left(\mathbf{5}^{\prime} \mathbf{-} \mathbf{3}^{\prime} \mathbf{)}\right.$ \\
\hline CD31 & TGGAAACCAACAGCCATTACG & GGGAGCCTTCCGTTCTCTTG \\
VEGFA & CAGGAGCCTGGCCATCAA & CCTCTTCTTCCACCACTGTGTCT \\
VEGFR-2 & TGGTGGCTCAGGACGTTGA & TTCCCCTTTCTCCTCCGTTT \\
GAPDH & GGTGGACCTCATGGCCTACA & CAGCAACTGAGGGCCTCTCT \\
\hline
\end{tabular}

PCR data analysis was performed using StepOne ${ }^{\mathrm{TM}}$ software v2.0.2 (Thermo Fisher Scientific, Waltham, US). Relative quantitation of gene expression was assessed between quantity of target gene and GAPDH using relative standard curve method.

\section{Statistical analysis}

IBM SPSS 24.0 (IBM Crop, Armonk: NY, USA) was used for data analyses. Relative expression levels of genes were expressed as mean \pm standard deviation and statistically analyzed using independent-samples $t$-test. $\mathrm{P}<0.05$ was considered statistically significant.

\section{Results}

\section{Clinical observation}

Gross observation of defect site showed normal healing of surgical wounds in majority (28/32) of the animals in ZA and Control groups (Additional file 1: Figs. S1, S2). No soft tissue fenestration or bone exposure was found in femur. Delayed soft tissue healing was found in both ZA group (3 in mandible, 4 in maxilla) and control group ( 1 in mandible, 2 in maxilla), while exposed bone was found only in the ZA group ( 1 in maxilla, 2 in mandible) (Table 2).

\section{Real-time PCR}

All the animals were sacrificed for real-time PCR analysis to determine the relative mRNA expression levels of angiogenesis markers CD31, VEGFR-2 and VEGFA. Serial two-time dilution of the cDNA was applied for RTPCR. Standard curve for each target gene was obtained (Additional file 1: Figs. S3, S4).

The fold-difference of quantity in ZA group relative to control group was shown in Table 3. In femur defect, the expression of these angiogenetic markers showed no significant difference between control groups and ZA-treated groups. In the mandible, 1.12fold of VEGFA expression was found in short-term ZAtreated group compared with corresponding control

Table 2 Anatomy observation in the mandibular, maxillary extraction site and femur defect

\begin{tabular}{|c|c|c|c|c|c|c|c|}
\hline & \multirow[t]{2}{*}{$\mathbf{N}$} & \multicolumn{2}{|l|}{ Femur } & \multicolumn{2}{|l|}{ Mandible } & \multicolumn{2}{|l|}{ Maxilla } \\
\hline & & $\begin{array}{l}\text { Soft tissue } \\
\text { fenestration }\end{array}$ & Exposed bone & $\begin{array}{l}\text { Soft tissue } \\
\text { fenestration }\end{array}$ & Exposed bone & $\begin{array}{l}\text { Soft tissue } \\
\text { fenestration }\end{array}$ & Exposed bone \\
\hline Treatment & 16 & 0 & 0 & $3(19 \%)$ & $2(13 \%)$ & $4(25 \%)$ & $1(6 \%)$ \\
\hline ZA-s & 8 & 0 & 0 & $1(13 \%)$ & 0 & $3(38 \%)$ & 0 \\
\hline ZA-m & 8 & 0 & 0 & $2(25 \%)$ & $2(25 \%)$ & $1(13 \%)$ & $1(13 \%)$ \\
\hline Control & 16 & 0 & 0 & $1(6 \%)$ & 0 & $2(13 \%)$ & 0 \\
\hline$C-s$ & 8 & 0 & 0 & $1(13 \%)$ & 0 & $1(13 \%)$ & 0 \\
\hline$C-m$ & 8 & 0 & 0 & 0 & 0 & $1(13 \%)$ & 0 \\
\hline
\end{tabular}


Table 3 The fold-difference between ZA-treated group normalized target and control group normalized target

\begin{tabular}{|c|c|c|c|c|c|c|}
\hline \multirow[t]{2}{*}{ Targets } & \multicolumn{2}{|l|}{ Femur } & \multicolumn{2}{|l|}{ Mandible } & \multicolumn{2}{|l|}{ Maxilla } \\
\hline & Short-term & Medium-term & Short-term & Medium-term & Short-term & Medium-term \\
\hline \multicolumn{7}{|l|}{ CD31 } \\
\hline Fold ${ }^{\mathrm{a}}$ & $1.02 \pm 0.21$ & $1.15 \pm 0.79$ & $0.69 \pm 0.40$ & $2.39 \pm 1.13$ & $1.32 \pm 0.97$ & $2.40 \pm 0.67$ \\
\hline p & 0.880 & 0.770 & 0.270 & 0.230 & 0.590 & 0.048 \\
\hline \multicolumn{7}{|l|}{ VEGFA } \\
\hline Fold & $0.99 \pm 0.15$ & $1.28 \pm 0.61$ & $1.12 \pm 0.43$ & $1.45 \pm 0.18$ & $1.33 \pm 0.30$ & $0.41 \pm 0.10$ \\
\hline$p$ & 0.120 & 0.820 & 0.016 & 1.000 & 0.060 & 0.029 \\
\hline \multicolumn{7}{|l|}{ VEGFR-2 } \\
\hline Fold & $1.38 \pm 0.24$ & $0.87 \pm 0.42$ & $1.37 \pm 0.54$ & $1.19 \pm 0.10$ & $1.43 \pm 0.54$ & $0.73 \pm 0.14$ \\
\hline$p$ & 0.480 & 0.400 & 0.069 & 0.082 & 0.040 & 0.039 \\
\hline
\end{tabular}

Differences are considered significant at $\mathrm{p}<0.05$ and highlighted in bold

${ }^{\text {a }}$ Fold-difference of ZA-treated group relative to control group, all values are normalized to GAPDH expression

$(\mathrm{p}=0.016)$. Among medium-term groups, expression of CD31, VEGFA and VEGFR-2 were higher in ZAtreated group but without statistically significance. In the maxilla, short-term ZA group expressed 1.43-fold of VEGFR-2 expression relative to the corresponding control $(\mathrm{p}=0.04)$. While medium-term ZA group showed 2.4-fold of CD31 expression compared with control $(\mathrm{p}=0.048)$. The expressions of VEGFA and VEGFR-2 were down-regulated in medium-term ZA group, in which 0.41 -fold of VEGFA $(\mathrm{p}=0.029)$ and 0.73 -fold of VEGFR-2 $(\mathrm{p}=0.039)$ lower than their corresponding control (Additional file 1: Fig. S5).

\section{Discussion}

MRONJ is realized as a pathological osteonecrosis condition of craniofacial skeleton with significant symptom of bone exposure after administration of antiresorptive agents or anti-angiogenesis drugs despite the existence of radiation treatment history. In our recent study, which used the same animal model, found that zoledronic acid treatment had a site-specific effect on surgically treated jawbones versus long bones, where necrosis occurred only in the jaw. This could be explained by the theory that Bps were considered as more preferentially deposited in skeleton structures with high turnover so that jawbones were more likely to be affected by these drugs. At the same time, osteonecrosis occurred more frequently at the site of the mandibles than maxillae since blood supply could play an important role to the metabolic activity [21]. The present study demonstrated similar result. No soft tissue fenestration or bone exposure was found in femur. Exposed bone was found only in the ZA treated animals, 1 out of 16 rats (6\%) in the maxillary extraction site, and 2 out of $16(13 \%)$ in the mandibular extraction site, indicating positive relevance of $\mathrm{ZA}$ treatment and osteonecrosis in jawbones.

The development of MRONJ was realized as multifactorial including osteoclast suppression, immune alteration, macrophage change, anti-angiogenesis, cell accumulation et al. In this study, we focused on the research of angiogenesis expression of animal model with BPs treatment. According to previous studies, it had been well known that BPs decrease endothelial proliferation which may influence angiogenesis [22, 23]. A micro-CT study performed by our group on the same rat model revealed that the ZA treatment had no significant effect on the microvasculature structure in the surgical healing sites of the jawbones and femur. A tendency of decreased vessel density and vessel number in ZA-treated group was found, but no statistical difference [24]. Inconsistent with the micro-CT findings, the present study found the expression of CD31, VEGFA and VEGFR-2 in the ZA treated group was slightly higher than that in the control group in femur, but no statistical significance. Similar results were found in the mandible, with the exception that VEGFA expression in the short-term ZA group was significantly higher (1.12-fold) when compared to the control group. The result indicated that in this study, the administration of ZA did not affect blood vessel formation. This was also reported by other studies which claimed normal angiogenesis in ONJ lesions [25, 26]. The upregulated tendency of angiogenetic markers in the femur and mandible, which is inconsistent with previous in vitro, animal and clinical studies, might be a compensatory result of the antiangiogenic potency of BPs [16-18]. 
Interestingly, the present study demonstrated a different expression pattern of the angiogenetic markers in maxilla. The gene expression of VEGFA and VEGFR-2 in ZA-treated group is significantly down-regulated compared with control. The unexpected finding in maxilla might be attributed to the difference in anatomical and angiogenic structures between mandible and femur, however, the down-regulated expression of the angiogenic factors in maxilla in the ZA treated group does not result in a compromised angiogenesis [27, 28].

Overall, the expression of the angiogenic factors in femur and mandible is not significantly affected by BPs treatment, whereas it is significantly decreased in response to surgical intervention in maxilla. Given the higher occurrence of MRONJ in mandible compared with that in maxillary bone, the down-regulated expression of angiogenic factor under ZA treatment may not be directly associated with the site-specific feature of MRONJ.

\section{Conclusions}

The ZA treatment does not change significantly the expression of the angiogenic factors in femur and mandible, but significantly downregulates the expression in maxilla in this rat model. The angiogenesis inhibition may contribute to the development of MRONJ but does not play a key role.

\section{Limitation}

It is noteworthy that this study was primarily focused on the assessment of bone exposure and osteonecrosis, however, with the limitation of the known factors, more investigations are required for more understanding of the underlying mechanisms responsible for the markers of angiogenesis in soft tissue such as gums of the animals treated with zoledronic acid.

\section{Abbreviations}

BPs: Bisphosphonates; CD31/PECAM-1: Cluster of differentiation 31/platelet endothelial cell adhesion molecule-1; cDNA: Complementary deoxyribonucleic acid; ECs: Extracellular space; GAPDH: Glyceraldehyde 3-phosphate dehydrogenase; MRONJ: Medication-related osteonecrosis of the jaws; RT -PCR: Real-time polymerase chain reaction; SD-rat: Sprague-Dawley rat; VEGF: Vascular endothelial growth factor; VEGFA: Vascular endothelial growth factor A; VEGFR-2: Vascular endothelial growth factor receptor-2; ZA: Zoledronic acid.
\end{abstract}

\section{Supplementary Information}

The online version contains supplementary material available at https://doi. org/10.1186/s13104-021-05900-5.

Additional file 1: Figure S1. Gross observation of healing of the extraction site on right maxillary first molar in ZAtreated groups and control groups. ZAs: Delayed soft tissue healing could be observed (yellow arrow); ZAm: Suspicious of bone exposure on the site of the extraction socket (yellow arrow); ZAl, Cs, Cm Cl: Optimal healing of soft tissue on the extraction site. Blue arrows indicate normal healing after tooth extraction. M2: Maxillary second molar. Figure S2. Gross observation of healing of the extraction site on right mandibular first molar in ZA-treated groups and control groups. ZAs: Delayed soft tissue healing on the extraction site (yellow arrow); ZAm: Suspicious infection and bone exposure on the extraction area (yellow arrow); ZAl: Suspicious bone exposure on the extraction site (yellow arrow); Cs: Slightly delayed healing of soft tissue (yellow arrow); Cm, Cl: Optimal healing of soft tissue on the extraction site (blue arrow). M2: Mandibular second molar. Figure S3. Amplification plot for housekeeping gene GAPDH (a) and target gene CD31 (c); Standard curve for GAPDH (b), $R^{2}=0.998$, and target gene CD31(d). $R^{2}=0.997 . R^{2}$ : correlation coefficient. Figure S4. Amplification plot for target gene VEGFA (a) and VEGFR-2 (c); Standard curve for VEGFA (b), $R^{2}=0.994$, and VEGFR-2 (d). $R^{2}=0.997$. $R^{2}$ : correlation coefficient. Figure $S 5$. Relative quantity of CD31, VEGFR-2 and VEGFA in bone biopsies (femur (a, $d, g$ ); mandible (b, e, h); and maxilla $(c, f, i)$ ) of 2 weeks and 4 weeks post-operation. Values are normalized to GAPDH expression. Difference is considered significant at ${ }^{*} p<0.05$

\section{Acknowledgements}

We appreciate the valuable technical assistance provided by the Laboratory Animal Unit, and Orthopaedic Research Centre, Department of Orthopaedics and Traumatology of the Li Ka Shing Faculty of Medicine, The University of Hong Kong.

\section{Authors' contributions}

Dr. JWL and Dr. JYW contributed equally to this work. JWL: results interpretation, manuscript drafting and revision; JYW: animal experiment, laboratory analysis and manuscript drafting; RQY: animal experiment, laboratory analysis; LH: manuscript revision; LWZ: study conception and design. All the authors agree to be accountable for all aspects of the work in ensuring that questions related to the accuracy or integrity of any part of the work are appropriately investigated and resolved. All authors read and approved the final manuscript.

Funding

The work is supported by the Seed Fund for Basic Research (201910159268), the University of Hong Kong.

\section{Availability of data and materials}

The datasets generated and analysed during the current study are available from the corresponding author on reasonable request.

\section{Declarations}

\section{Ethics approval and consent to participate}

This article does not contain any studies with human participants performed by any of the authors. All applicable international, national, and/or institutional guidelines for the care and use of animals are followed. The protocol of the animal experiment was approved by the Committee on Use Live Animal for Teaching and Research, The University of Hong Kong (CULATR 3775-15).

\section{Consent for publication}

Not applicable.

\section{Competing interests}

The authors declare that they have no competing interests.

\section{Author details}

${ }^{1}$ Discipline of Oral and Maxillofacial Surgery, Faculty of Dentistry, The University of Hong Kong, Hong Kong SAR, China. ' Department of Orthodontics, The First Affiliated Hospital of Zhengzhou University, Zhengzhou, Henan Province, China.

Received: 19 October 2021 Accepted: 23 December 2021

Published online: 10 January 2022 


\section{References}

1. Marx RE, Sawatari Y, Fortin M, et al. Bisphosphonate-induced exposed bone (osteonecrosis/osteopetrosis) of the jaws: risk factors, recognition, prevention, and treatment. J Oral Maxillofac Surg. 2005;63(11):1567-75.

2. Ferrara N, Gerber HP, LeCouter J. The biology of VEGF and its receptors. Nat Med. 2003;9(6):669-76.

3. Koerdt S, Ristow O, Wannhoff A, et al. Expression of growth factors during the healing process of alveolar ridge augmentation procedures using autogenous bone grafts in combination with GTR and an anorganic bovine bone substitute: an immunohistochemical study in the sheep. Clin Oral Invest. 2014;18(1):179-88.

4. Zelzer $\mathrm{E}$, Olsen BR. Multiple roles of vascular endothelial growth factor (VEGF) in skeletal development, growth, and repair. Curr Top Dev Biol. 2005;65:169-87.

5. Marini M, Bertolai R, Manetti M, et al. A case of mandible hypoplasia treated with autologous bone graft from mandibular symphysis: expression of VEGF and receptors in bone regeneration. Acta Histochem. 2016;118(6):652-6.

6. Clarkin CE, Gerstenfeld LC. VEGF and bone cell signalling: an essential vessel for communication? Cell Biochem Funct. 2013;31(1):1-11.

7. Mayr-Wohlfart U, Waltenberger J, Hausser H, et al. Vascular endothelial growth factor stimulates chemotactic migration of primary human osteoblasts. Bone. 2002;30(3):472-7.

8. Detmar M. Molecular regulation of angiogenesis in the skin. J Invest Dermatol. 1996;106(2):207-8.

9. Ferrara N. Vascular endothelial growth factor: molecular and biological aspects. Curr Top Microbiol Immunol. 1999:237:1-30.

10. Shibuya M, Luo JC, Toyoda M, et al. Involvement of VEGF and its receptors in ascites tumor formation. Cancer Chemother Pharmacol. 1999;43(Suppl):S72-77.

11. Schmidt T, Carmeliet P. Angiogenesis: a target in solid tumors, also in leukemia? Hematology Am Soc Hematol Educ Program. 2011;2011:1-8.

12. Raica M, Cimpean AM, Anghel A. Immunohistochemical expression of vascular endothelial growth factor (VEGF) does not correlate with microvessel density in renal cell carcinoma. Neoplasma. 2007;54(4):278-84.

13. Sato J, Segami N, Nishimura M, et al. Correlation between the arthroscopic diagnosis of synovitis and microvessel density in synovial tissues in patients with internal derangement of the temporomandibular joint. J Craniomaxillofac Surg. 2003;31(2):101-6.

14. Kauhanen S, Salmi A, von Boguslawski K, et al. Satellite cell proliferation, reinnervation, and revascularization in human free microvascular muscle flaps. J Surg Res. 2003;115(2):191-9.

15. Ishtiaq S, Edwards S, Sankaralingam A, et al. The effect of nitrogen containing bisphosphonates, zoledronate and alendronate, on the production of pro-angiogenic factors by osteoblastic cells. Cytokine. 2015;71(2):154-60.

16. Ohlrich EJ, Coates DE, Cullinan MP, et al. The bisphosphonate zoledronic acid regulates key angiogenesis-related genes in primary human gingival fibroblasts. Arch Oral Biol. 2016;63:7-14.

17. Thumbigere-Math V, Michalowicz BS, Hughes PJ, et al. Serum markers of bone turnover and angiogenesis in patients with bisphosphonate-related osteonecrosis of the jaw after discontinuation of long-term intravenous bisphosphonate therapy. J Oral Maxillofac Surg. 2016;74(4):738-46.

18. Borke JL, McAllister B, Harris T, et al. Correlation of changes in the mandible and retina/choroid vasculature of a rat model of BRONJ. J Craniomaxillofac Surg. 2015. https://doi.org/10.1016/j.jcms.2015.05.021.

19. Kim SM, Eo MY, Cho YJ, et al. Wound healing protein profiles in the postoperative exudate of bisphosphonate-related osteonecrosis of mandible. Eur Arch Otorhinolaryngol. 2017;274(9):3485-95.

20. Nastro Siniscalchi E, Cutroneo G, Catalfamo L, et al. Immunohistochemial evaluation of sarcoglycans and integrins in gingival epithelium of multiple myeloma patients with bisphosphonate-induced osteonecrosis of the jaw. Oncol Rep. 2010;24(1):129-34.

21. Wang JY, Huo L, Yu RQ, et al. Skeletal site-specific response of jawbones and long bones to surgical interventions in rats treated with zoledronic acid. Biomed Res Int. 2019;2019:5138175.

22. Allegra A, Alonci A, Penna G, et al. Bisphosphonates induce apoptosis of circulating endothelial cells in multiple myeloma patients and in subjects with bisphosphonate-induced osteonecrosis of the jaws. Acta Haematol. 2010;124(2):79-85.
23. Allegra A, Oteri G, Nastro E, et al. Patients with bisphosphonates-associated osteonecrosis of the jaw have reduced circulating endothelial cells. Hematol Oncol. 2007:25(4):164-9.

24. Wang JY, Yu RQ, Huo L, et al. Comparison of vascular morphometry in jawbones and long bones: micro-CT study in a rat model treated with zoledronic acid. Biomed Res Int. 2021;2021:6651318.

25. Khan AA, Morrison A, Hanley DA, et al. Diagnosis and management of osteonecrosis of the jaw: a systematic review and international consensus. J Bone Miner Res. 2015:30(1):3-23.

26. Hansen T, Kunkel M, Weber A, et al. Osteonecrosis of the jaws in patients treated with bisphosphonates-histomorphologic analysis in comparison with infected osteoradionecrosis. J Oral Pathol Med. 2006;35(3):155-60.

27. Katsarelis H, Shah NP, Dhariwal DK, et al. Infection and medication-related osteonecrosis of the jaw. J Dent Res. 2015;94(4):534-9.

28. Russell RG, Watts NB, Ebetino FH, et al. Mechanisms of action of bisphosphonates: similarities and differences and their potential influence on clinical efficacy. Osteoporos Int. 2008;19(6):733-59.

\section{Publisher's Note}

Springer Nature remains neutral with regard to jurisdictional claims in published maps and institutional affiliations.

Ready to submit your research? Choose BMC and benefit from

- fast, convenient online submission

- thorough peer review by experienced researchers in your field

- rapid publication on acceptance

- support for research data, including large and complex data types

- gold Open Access which fosters wider collaboration and increased citations

- maximum visibility for your research: over 100M website views per year

At $\mathrm{BMC}$, research is always in progress.

Learn more biomedcentral.com/submissions 